\title{
A Case of Type II Cystinuria
}

\author{
Ken Mryagi, M D* and Fukuichi Nakada, M D**
}

\begin{abstract}
A case of cystinuria with hyperuricaemia was reported. The patient had had four times operations for vesical and ureteral calculi and there still remained two prostate stones. The serum concentration of basic amino acids was normal but urinary concentration of cystine was 300 times, lysine 52 times and arginine 5.7 times of normal value. After oral cystine load of $0.05 \mathrm{~m} \mathrm{moles} / \mathrm{kg}$ body weight, the plasma cystine level of the case showed decrease as contrasted to increase in normal subjects. The urinary cystine concentration of heterozygote of this case was $138 \mathrm{mg} / \mathrm{g}$ creatinine, and both lysine and cystine were increased. From these results, the present case was identified as type II cystinuria. The reabsorption rate of basic amino acids was studied. As contrasted to the rate of more than $90 \%$ in normal, that of lysine and arginine in this case were $47.4 \%$ and $67.2 \%$, respectively. Interestingly, the reabsorption rate of cystine in this case was $-117 \%$ as contrasted to $+99.4 \%$ in normal subjects. This means that cystine excreted in urine was greater than that filtered through glomerulus, and suggests the possible secretion of cystine from renal tubules. The basic disorder of cystinuria will be cellular disturbance of cystine transport expressed as cystine secretion.
\end{abstract}

Key Words: Cystinuria, Amino acid, Reabsorption.

Among the patients who are suffering from repeated calculus formation in urinary tract, it is not seldom to find cystinuria. The main cause for the stone formation in this disease is believed to be the precipitation in urine, especially in acidic urine, of cystine which is excreted in excess amounts because of the inborn error of renal tubular reabsorption.

The authors have recently experienced a case of type II cystinuria with hyperuricaemia who had had four times operations for vesical and ureteral calculus. This case is reported together with results suggesting the possible secretion of cystine from renal tubules.

\section{CASE REPORT}

Case.-K.N. 69 years old man.

Chief complaint.-Abdominal pains in ileo-

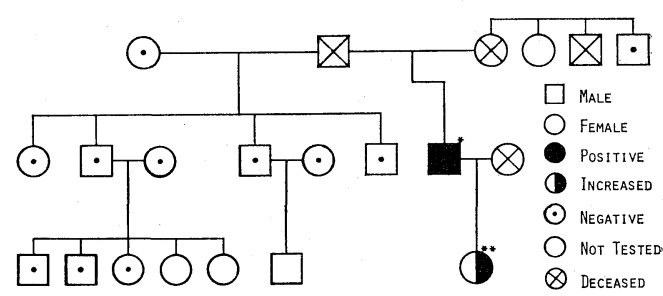

Fig. 1. Family tree.

* Present case ** Heterozygote

coecal region.

Past history.--Not remarkable except for chyluria in 1954, and calculus formation described below.

Family history.--As shown in Fig. 1, his daughter showed elevated cystine excretion. The urine of other member of his family have all shown negative sodium nitroprusside test. His mother died of puerperal fever

\footnotetext{
* From Clinical Pathology, College of Health Sciences, University of the Ryukyus, Naha, Okinawa

** Department of Biochemistry, College of Health Sciences, University of the Ryukyus, Naha, Okinawa Received for publication June 3, 1977.

Reprint request to: Ken Miyagi, University of the Ryukyus, College of Health Sciences, 585 Yogi, Naha City, Okinawa, MZ902, Japan.
} 
at age 24 and his father of possible liver cirrhosis at age 85 .

Present illness.-The patient had been in good health until Spring of 1949 when he had sudden mid-lower abdominal pain radiating to testis and penis, and had sometimes experienced a retention of urine. There had been no macrohematuria. Under the diagnosis of vesical calculus, he had had an operation. The second episode was in Summer of 1970. The signs and symptoms were the same as before. In both instances, the stone had been brown in colour, cashew nut shape and a size of fingertip. The third episode was in Summer of 1971 when he had had sudden right lower abdominal pain. There had been normal urination and no macrohematuria. Operation had been done under the diagnosis of right ureteral calculus. The stone had had a mixture of brown and white in colour, irregular shape and a size of soybean. The fourth episode was in Autumn of 1973. Two weeks before admission, he began to have a dull abdominal pain in the right lower quadrant. Neither macrohematuria, constipation nor diarrhea were present. For three days, the pain had changed to calculus-colic type and he visited our out-patient clinic.

Physical examination.--The patient was somewhat smaller than normal stature $(158 \mathrm{~cm})$, normally-nourished (58 kg), appeared acutely ill and complained of colic abdominal pain in the right lower quadrant. His hair and face were normal. Neither fever, jaundice nor anemia were present. Blood pressure was $110 / 60 \mathrm{mmHg}$. The lung and heart were negative. No hepatosplenomegalia was present and the kidnies were not palpable. There were spontaneous pain at right lower quadrant and right low back pain, and was localized tenderness at the right lumber region. No edema on the extremities. Neuropsychiatric examinations were negative. No signs suggestive of gout were present.

Laboratory findings. - Urinalysis showed $\mathrm{pH}$ 7.0 ; the sediment contained 3 to 5 red cells and hexagonal crystals characteristic for cystine per high power field. The routine hematological examination, liver function tests and lipid metabolites were normal. PSP excretion test was 22.9, 23.7, 18.8 and $13.3 \%$ for $15,30,60$ and $120 \mathrm{~min}$, respectively. BUN was 13 to $22 \mathrm{mg} / \mathrm{dl}$ and serum uric acid 8.0 to $8.7 \mathrm{mg} / \mathrm{dl}$.

A scout film of abdomen revealed a calcification at right lumber region and other two small stones at the pubic symphysis. Intravenous pyelography disclosed right hydronephrosis, and the calculus at the right lumbar region was confirmed to be an ureteral stone (Fig. 2).

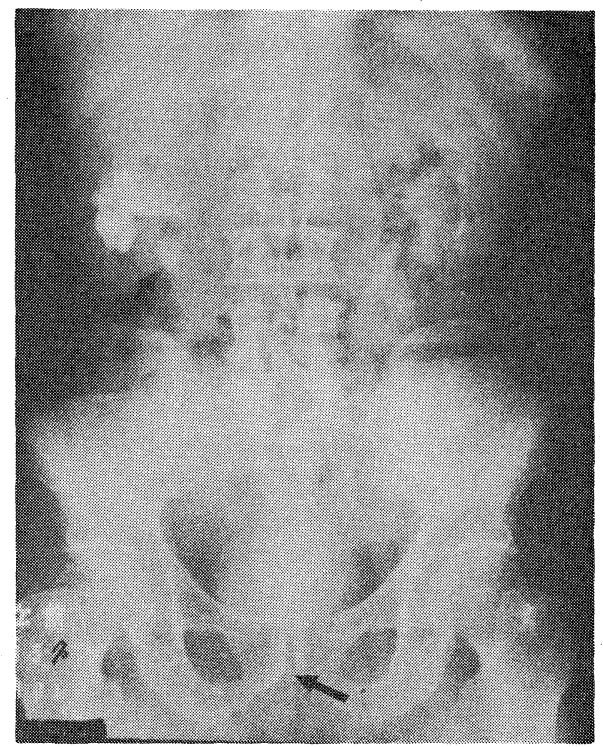

Fig. 2. X-ray film of intravenous pyelography. Arrow indicates an ureteral calculus and prostate calculi.

Course.-After the operation for ureteral stone, the solubilization of urinary cystine was controlled with 200 to $600 \mathrm{mg} /$ day of D-penicillamine ${ }^{1,2)}$, and hyperuricaemia with 100 to $200 \mathrm{mg} /$ day of allopurinol. Both drugs were administered for about three years but no side effects have been observed.

\section{IDENTIFICATION AS TYPE II CYSTINURIA}

\section{(1) Oral cystine load test}

Rosenberg et $\mathrm{al}^{3)}$ have reported three genetically distinct types in cystinuria. According to their procedure of oral cystine 
load test, the present case was intended to be classified into the subtype.

A $0.5 \mathrm{~m}$ moles of cystine per $\mathrm{kg}$ of body weight was administered orally. The serum cystine concentration of before, 1, 2 and 3 hours after load was determined. It should be noted that the oxidation of cysteine to cystine was carefully avoided ${ }^{4}$. Deproteinization was carried out by addition of $5 \mathrm{ml}$ of $1 \%$ pycric acid to $1.0 \mathrm{ml}$ of serum. After centrifugation, the picric acid in the supernatant solution was completely removed by passing through the column of Dowex 1-X8. The quantitative analysis of amino acids was carried out by an automatic amino acid analyzer JEOL JLG-6AH. D-penicillamine therapy was discontinued from 1 week before the experiment.

The results of serum cystine concentration before and after oral cystine load to this patient and to a 40 years old healthy man were shown in Fig. 3. As contrasted to the increase in serum cystine level in the normal control, the present case showed decrease after cystine load. It is reported that type III cystinuria alone showed increase as contrasted to decrease in type I and II in the serum cystine level ${ }^{3}$. Thus, the present case was supposed to be either type I or II.

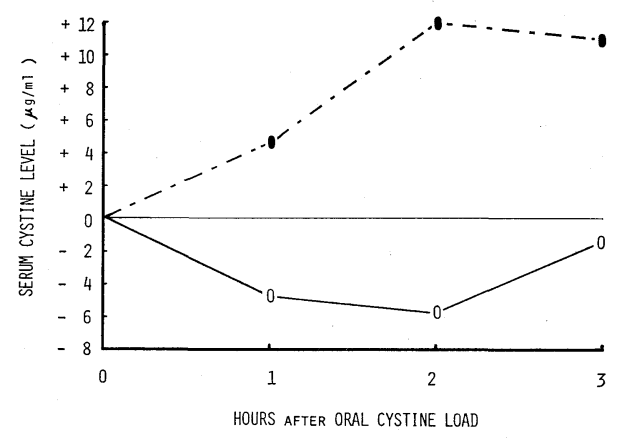

Fig. 3. Serum cystine concentration in normal and the present case after oral cystine load.

- - normal $\bigcirc-\bigcirc$ present case

\section{(2) Urinary cystine level of heterozygote}

According to the observation of Rosenberg et $\mathrm{al}^{3)}$, the three types of cystinuria can be roughly classified by urinary cystine concentration of heterozygotes. However, there were overlapping between type I and III, and between type II and III. There was no overlap between type I and II, and they can be differentiated.

The urine of patient's daughter showed positive nitropluscide test, and the cystine concentration in the first void urine of several days was determined by the method of Shinohara et $\mathrm{al}^{5)}$. The mean value was 138 $\mathrm{mg} / \mathrm{g}$ creatinine. This was clearly higher than the upper limit of normal $(50 \mathrm{mg} / \mathrm{g}$ creatinine) and upper limit of type I heterozygote $(60 \mathrm{mg} / \mathrm{g}$ creatinine), and overlapped with heterozygotes of type II and III. This result suggests the daughter of present case to be either the type II or III.

\section{(3) Urinary amino acid excretion pattern}

As a normal control, three healthy men aged 23, 40 and 55 were determined for the amino acids excretion in the fasted urine of early morning. When the mean value of them was expressed as $100 \%$, the percent increase of urinary amino acid in the patients and heterozygote was shown in Fig. 4. It is reported that, in the urine of cystinuria, cystine and other basic amino acids, namely, lysine, arginine and ornithine were increased. In the present case too, cystine,

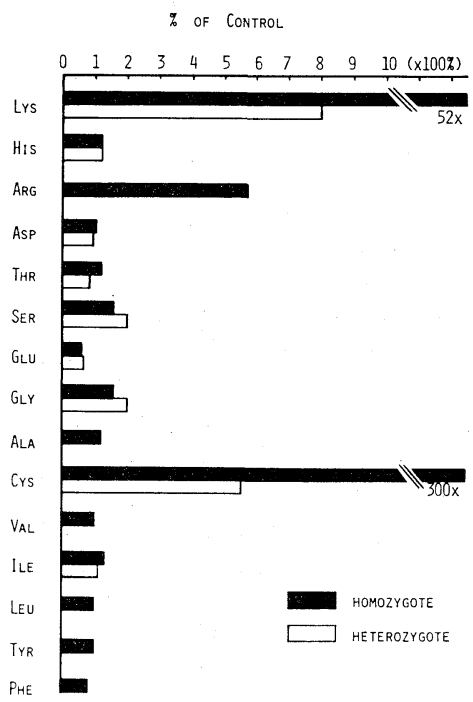

Fig. 4. Urinary amino acids excretion pattern of the present case and its heterozygote.

Jap J Med Vol 17, No 1 (January 978) 
lysine and arginine were increased to 300 , 52 and 5 times normal, respectively. Other than cystine, the increased excretion of lysine was outstanding.

The amino acid excretion pattern in the urine of heterozygotes is reported significantly different ${ }^{6)}$. The levels of cystine and lysine are reported normal in the urine of type I heterozygotes, but increased in type II and III. The heterozygote of our case showed increase of lysine to 8 times normal and of cystine to 5.5 times normal as shown in Fig. 4. This amino acid excretion pattern also suggests the possibility that the present case is type II or III cystinuria.

From the above mentioned results of oral cystine load, urinary cystine level of heterozygote and urinary amino acid excretion pattern of heterozygote, the present case was identified as type II cystinuria.

\section{POSSIBLE SECRETION OF CYSTINE}

Urinary excretion of cystine in this case was increased to as much as 300 times of normal value (Fig. 4). To understand the mechanism of the increased urinary excretion of amino acids in this disease, it will be of a considerable interest to examine the reabsorption rate of amino acids.

Both. 40 years old man in good health and the patient were fasted until the end of experiment. A $300 \mathrm{ml}$ of water was ingested. One hour later they urinated completely and the urine was discarded. Then, another one hour urine was collected and blood was drawn at the mid point of urine collection. The creatinine concentration in blood and urine sample was determined and GFR was estimated from creatinine clearance. From GFR and cystine concentration in the serum, the amount of cystine filtered through glomerulus in one hour was calculated. The cystine excreted in urine in one hour was calculated from urinary cystine concentration and one hour urine volume. The excreted cystine was subtracted from filtered cystine, and this was divided by filtered cystine. This was designated as cystine reabsorption rate and expressed as \% reabsorption. The reabsorption rate of other amino acids were similarly calculated. The results were shown in Table 1.

As contrasted to more than $90 \%$ in the control, the reabsorption rate of lysine and arginine in the present case was 47.4 and $67.2 \%$, respectively. Other amino acids indicated in Fig. 4 were all more than 90\% in both normal and the present case. It would be noted that the reabsorption rate of cystine in this case was $-117 \%$ as contrasted to $+99.4 \%$ in normal control. The GFR of this case was somewhat lower than normal, but even when the reabsorption rate were calculated on the basis of normal GFR $(110.26 \mathrm{ml} / \mathrm{min})$, it was still $-61 \%$. This means that the cystine excreted in urine exceeds the amount filtered through glomerulus and suggests that the cystine was secreted from renal tubules in this case.

\section{DISCUSSION}

Cystinuria is an inherited disease of amino acid transport disturbance, characterized clinically by calculus formation in urinary tract. The main cause for stone formation is crystallization of cystine which

Table 1. Reabsorption rate of amino acids in normal and the present case.

\begin{tabular}{|c|c|c|c|c|c|c|c|c|}
\hline & & $\begin{array}{l}\text { Serum A.A.* } \\
\text { Concentration } \\
\mathrm{mg} / \mathrm{d} \mathrm{l}\end{array}$ & $\begin{array}{l}\text { Creatinine } \\
\text { Clearance } \\
\mathrm{m} 1 / \mathrm{min}\end{array}$ & $\begin{array}{l}\text { A.A.* } \\
\text { filtered } \\
\mathrm{mg} / 60 \mathrm{~min}\end{array}$ & $\mid \begin{array}{c}\text { Urinary A.A.* } \\
\text { Concentration } \\
\mathrm{mg} / \mathrm{d} \mathrm{l}\end{array}$ & $\begin{array}{l}\text { Urine } \\
\text { volume } \\
\mathrm{m} 7 / 60 \mathrm{~min}\end{array}$ & $\begin{array}{l}\text { Total A.A.* } \\
\text { excreted } \\
\mathrm{mg} / 60 \mathrm{~min}\end{array}$ & $\begin{array}{c}\text { Reabsorption } \\
\text { Rate } \\
\%\end{array}$ \\
\hline \multirow{3}{*}{ Present Case } & Cys & 0.1610 & 81.749 & 7.8970 & 4.7608 & 360 & 17.1389 & -117.0 \\
\hline & Lys & 2.7264 & 81.749 & 133.7283 & 19.5570 & 360 & 70.4025 & +47.4 \\
\hline & Arg & 0.5049 & 81.749 & 24.7650 & 2.2545 & 360 & 8.1162 & +67.2 \\
\hline \multirow{3}{*}{ Norma 7} & Cys & 0.1970 & 110.260 & 13.0327 & 0.0312 & 265 & 0.0827 & +99.4 \\
\hline & Lys & 5.4251 & 110.260 & 358.9029 & 0.7500 & 265 & 1.9875 & +99.4 . \\
\hline & Arg & 7.0784 & 110.260 & 71.3426 & 2.4509 & 265 & 6.4949 & +90.9 \\
\hline
\end{tabular}

* A.A. = Amino Acids. 
is excreted in excessive amount in urine. The high incidence of stone formation in acidic urine can be accounted for by the insolubility of cystine in acidic solution ${ }^{7}$. Meloni et $\mathrm{al}^{8)}$ reported that the incidence of stone formation was higher in cystinuria with hyperuricaemia than in the case without it. The hyperuricaemia in our case undoubtedly influenced the calculus formation so often as to have four times operations for vesical and ureteral stones.

As shown in Fig. 4, the present case excreted a large amount of basic amino acids. Since there was an impairment of PSP excretion in this case, one may postulate that the excessive excretion of basic amino acids is secondary to amino aciduria due to renal insufficiency. However, in the secondary amino aciduria, it will be very rare that only basic amino acids are excreted excessively. Many other amino acids should also be increased in minor degree ${ }^{9)}$.

The excessive urinary basic amino acids are not due to increase in the serum. As shown in Table 2, the present case revealed normal serum amino acid levels. This point is different from other amino aciduria as for example phenylketonuria.

Recently, two mechanisms have been postulated for the reabsorption of amino $\operatorname{acids}^{11)}$. One is a group specific system; it has a high-capacity but low-affinity for its substrates and there is a competitive inhibition. The other is a substrate specific system; it has a low-capacity but highaffinity for its substrates and there is little response to competitive inhibition. If this hypothesis were adopted to the present case, the excessive urinary excretion of lysine and arginine will be a result of competitive inhibition by a large excess of cystine present in the urine. Namely, the reabsorption of lysine and arginine was inhibited competitively. For the reabsorption of these amino acids, only the substrate specific system which has a small capacity was left, and they were excreted in excess amounts. The decrease in reabsorption rate of lysine and arginine in this case (see Table 1) sup-
Table 2. Serum amido acid level of normal and the present case.

\begin{tabular}{l|c|c|c} 
& Norma & $\begin{array}{c}\text { Present } \\
\text { Case } \\
\mathrm{mg} / \mathrm{d} 1\end{array}$ & $\begin{array}{c}\text { Normal Range } \\
\text { mg/d }\end{array}$ \\
\hline Lys & 5.43 & 2.73 & $1.21-3.48$ \\
His & 1.71 & 1.31 & $0.49-1.66$ \\
Arg & 1.08 & 0.50 & $0.37-2.63$ \\
Asp & 0.16 & 0.05 & $0.00-0.72$ \\
Thr & 7.08 & 7.53 & $0.94-2.93$ \\
Ser & 0.64 & 1.62 & $0.68-2.03$ \\
Glu & 1.03 & 1.04 & $0.21-2.28$ \\
Gly & 1.87 & 2.00 & $0.90-4.16$ \\
Ala & 3.38 & 3.38 & $1.87-5.89$ \\
Cys & 0.20 & 0.16 & $0.20-3.37$ \\
Val & 2.67 & 2.65 & $1.36-3.71$ \\
Ile & 0.91 & 0.70 & $0.46-1.28$ \\
Leu & 1.66 & 1.06 & $0.93-2.30$ \\
Tyr & 0.94 & 0.92 & $0.39-1.58$ \\
Phe & 0.87 & 0.55 & $0.61-1.29$
\end{tabular}

* The normal values were mean of 23,40 and 55 years old males.

** The normal range were made by taking the lowest and highest values in reference (10).

ports the above hypothesis.

This explanation does not conflict with the report of Fox et $\mathrm{al}^{122}$. They reported that, by the intravenous administration of lysine, the reabsorption of arginine was strongly inhibited in normal, while it was not inhibited in cystinuria. This will be explained as follows. In normal subject under physiological condition, arginine is completely reabsorbed with substrate specific and group specific systems, but under the condition of lysine being loaded greater than the large capacity of group specific systems, the reabsorption of arginine was inhibited competitively. On the other hand, in cystinuria, there is already an over-load by large excess of cystine and the reabsorption of arginine is already inhibited competitively. Thus, even if lysine were loaded, the arginine excretion did not increase more than already increased amount. Therefore, the inhibitory effect was not observed.

As mentioned above, the increased excretion of cystine plays an important role for both clinical problem of calculus forma- 
tion and the pathophysiological problem of excessive excretion of basic amino acids. A very interesting phenomena is that the reabsorption rate of cystine was $-117 \%$ in this case (Table 1). Suppose that there were no reabsorption, the percent reabsorption should be $0 \%$. The negative reabsorption means that the amount of cystine excreted in urine was larger than the amount filtered through glomerulus. This suggests that cystine was secreted from renal tubules. Frimpter et al $^{13)}$ also reported that the reabsorption rate of cystine was $-50 \%$ in human cystinuria ${ }^{14)}$. In the in vitro experiments using rat kidney cortex slices, the results were reported suggesting the secretion of cystine and cysteine ${ }^{15)}$.

In general the secretion from renal tubules occurs only when certain substance increased in serum excessively, except ammonia and other metabolites which are toxic or unnecessary to the body. It will be a specific abnormality to this disease that the amino acid which is necessary for human body is secreted without any increase in serum and without any organic damage of renal tubules. The investigation of the mechanism of this cystine secretion would be an interesting subject to be solved.

\section{CONCLUSION}

A case of cystinuria with hyperuricaemia was reported. He had repeated operations for vesical and ureteral stones, and was diagnosed as type II cystinuria from the results of oral cystine load and the urinary amino acid excretion pattern of heterozygote. The reabsorption rate of cystine in this patient calculated from creatinine clearance suggested the possible secretion of cystine from renal tubules.

\section{REFERENCES}

1) Crawhall JC, Scowen EF, Watts RWE : Effect of penicillamine on cystinuria. Brit Med J $1: 588,1963$.

2) Grawhall JC, Scowen EF, Watts RWE: Further observations on use of D-penicillamine in cystinuria. Brit Med J 1: 1411, 1964.

3) Rosenberg LE, Downing S, Durant JL: Cystinuria: Biochemical evidence for three genetically distinct diseases. J Clin Invest 45 : 365, 1966.

4) Brigham MP, Stein WH, Moore S: The concentration of cysteine and cystine in human blood plasma. J Glin Invest 39 : 1633, 1960.

5) Shinohara K, Padis LE: The determination of thiol and sulfide compounds, with special reference to cysteine and cystine. VII. Application of the modified phospho-18-tungstic acid method for the determination of cysteine, cystine, and ascorbic acid in urine. $\mathrm{J}$ Biol Chem 112: 709, 1935.

6) Rosenberg LE: Genetic heterogeneity in cystinuria. In: Amino acid metabolism and genetic variation (Nyhan WL, ed), McGrawHill, New York, p 341, 1967.

7) Dent UE, Senior B: Studies on the treatment of cystinuria. Brit J Urol $27: 317,1955$.

8) Meloni GR, Canary JJ : Cystinuria with hyperuricemia. JAMA 200: 169, 1967.

9) Wallis LA, Engle RL: The adult Fancony syndrome; To review eighteen cases. Amer J Med 22: 13, 1959.

10) Hamilton PB : Free amino acids in blood plasma of new born infants and adults. In : Handbook of biochemistry. Selected data for molecular biology (Sober HA, Harte RA, Sober EK, eds), Chemical Rubber Co, p B100, 1970.

11) Rosenberg LE, Segal S: Lysine transport in human kidney: Evidence for two systems. Science 155: 1426, 1967.

12) Fox M, Thier S, Rosenberg L : Ionic requirement for amino acid transport in the rat kidney cortex slices. Influence of the extracellular ions. Biochim Biophys Acta 79:167, 1964.

13) Frimpter GW, Horwith M, Furth E, Fellows RE, Thompson DD, Bass A, Schechter N : Inulin and endogenous amino acid renal clearances in cystinuria: Evidence for tubular secretion. J Glin Invest $41: 281,1962$.

14) Bovee KC, Thier SO, Rea C, Segal S : Renal clearance of amino acids in canine cystinuria. Metabolism 23 : 51, 1974.

15) Schwartzman L, Blair A, Segal S: A common renal transport system for lysine, ornithine, arginine and cystine. Biochem Biophys Res Comm 23 : 220, 1966. 